\title{
Below safety limits, every unit of glomerular filtration rate counts - reply letter
}

\author{
Carlotta Palumbo ${ }^{1,2}$, Alessandro Antonelli ${ }^{3,4}$ \\ ${ }^{1}$ Urology Unit, ASST Spedali Civili of Brescia, Brescia, Italy; ${ }^{2}$ Department of Medical and Surgical Specialties, Radiological Science and Public \\ Health, University of Brescia, Brescia, Italy; ${ }^{3}$ Department of Urology, Azienda Ospedaliera Universitaria Integrata, Verona, Italy; ${ }^{4}$ Department of \\ Surgery, Dentistry, Paediatrics and Gynaecology, University of Verona, Verona, Italy \\ Correspondence to: Alessandro Antonelli, MD. Professor, Department of Urology, Azienda Ospedaliera Universitaria Integrata, Verona, Italy; \\ Department of Surgery, Dentistry, Paediatrics and Gynaecology, University of Verona, Piazzale Aristide Stefani 1, 37126 Verona, Italy. Email: \\ alessandro_antonelli@me.com. \\ Provenance: This is an invited article commissioned by the Section Editor Dr. Xiao Li (Department of Urology, Jiangsu Cancer Hospital, Jiangsu \\ Institute of Cancer Research, Nanjing Medical University Affiliated Cancer Hospital, Nanjing, China). \\ Response to: Mistretta FA, Mazzone E, Knipper S, et al. Benefit of nephron sparing surgery translates into lower cancer specific mortality in patients \\ with localized renal cell carcinoma. Ann Transl Med 2018;6:S104. \\ Veccia A, Autorino R. Is there a relation between preserved renal function and oncological outcomes in patients undergoing partial nephrectomy for \\ renal cell carcinoma? Ann Transl Med 2018;6:S88
}

Submitted Nov 27, 2019. Accepted for publication Dec 06, 2019.

doi: $10.21037 / \mathrm{atm} .2019 .12 .54$

View this article at: http://dx.doi.org/10.21037/atm.2019.12.54

We value the editorials by Mistretta et al. (1) and Veccia et al. (2) on our recently published paper "Below safety limits, every unit of glomerular filtration rate counts: assessing the relationship between renal function and cancer-specific mortality in renal cell carcinoma" (3).

The authors (2) noted that our results seemed to be paradoxically in contrast with previous papers that showed that partial nephrectomy in the imperative setting was associated with higher recurrence, as well as higher cancerspecific mortality (CSM), even though it provided better preservation of renal function (4). Additionally, as reported by the authors, a previous study showed that patients with preoperatively impaired renal function tended to recover it after radical nephrectomy, more likely among patients with lower preoperative estimated glomerular filtration rate (eGFR) (5). Unfortunately, we were not able to specifically investigate the subset of patients, who were treated with partial nephrectomy due to an imperative indication. Nevertheless, sub-analyses according to type of surgery were performed. Specifically, a linear and inverse relationship between postoperative eGFR and CSM was found only below the breakpoint of $65 \mathrm{~mL} / \mathrm{min}$ for both partial and radical nephrectomy groups. It may be postulated that in patients for whom postoperative eGFR could be secured above this threshold, the role of surgery may be less determinant.

We also agree with the authors that the retrospective nature of our database may represent an important weakness of our study. Additionally, the multicenter source of data entailed a certain degree of heterogeneity in surgical techniques, peri-operative management, pathological assessment and follow-up schedules. Nonetheless, we attempted to overcome at least part of these issues by using multiple statistical approaches to assess the potential relationship between eGFR and CSM. All our models were adjusted for several confounders and all invariably supported our hypothesis. Nonetheless, we acknowledge that our results should be cautiously interpreted. Indeed, a multifaceted interplay exists among renal function, host and cancer (6), so that causal associations should not be attempted through a retrospective trial.

Finally, we agree with Mistretta et al. (1), as well as Veccia et al. (2), that additional studies are warranted to better elucidate the potential relationship between eGFR and CSM. Nonetheless, the results of our paper further highlight that preservation of renal function should be among priorities when planning surgery per kidney cancer. 


\section{Acknowledgments}

None.

\section{Footnote}

Conflicts of Interest: The authors have no conflicts of interest to declare.

Ethical Statement: The authors are accountable for all aspects of the work in ensuring that questions related to the accuracy or integrity of any part of the work are appropriately investigated and resolved.

\section{References}

1. Mistretta FA, Mazzone E, Knipper S, et al. Benefit of nephron sparing surgery translates into lower cancer specific mortality in patients with localized renal cell

Cite this article as: Palumbo C, Antonelli A. Below safety limits, every unit of glomerular filtration rate countsreply letter. Ann Transl Med 2020;8(4):137. doi: 10.21037/ atm.2019.12.54 carcinoma. Ann Transl Med 2018;6:S104.

2. Veccia A, Autorino R. Is there a relation between preserved renal function and oncological outcomes in patients undergoing partial nephrectomy for renal cell carcinoma? Ann Transl Med 2018;6:S88.

3. Antonelli A, Minervini A, Sandri M, et al. Below safety limits, every unit of glomerular filtration rate counts: assessing the relationship between renal function and cancer-specific mortality in renal cell carcinoma. Eur Urol 2018;74:661-7.

4. Adkins KL, Chang SS, Cookson MS, et al. Partial nephrectomy safely preserves renal function in patients with a solitary kidney. J Urol 2003;169:79-81.

5. Zabor EC, Furberg H, Lee B, et al. Long-term renal function recovery following radical nephrectomy for kidney cancer: results from a multicenter confirmatory study. J Urol 2018;199:921-6.

6. Iff S, Craig JC, Turner R, et al. Reduced estimated GFR and cancer mortality. Am J Kidney Dis 2014;63:23-30. 